\title{
PolySense: Augmenting Textiles with Electrical Functionality using In-Situ Polymerization
}

\author{
Cedric Honnet ${ }^{1}$, Hannah Perner-Wilson ${ }^{2}$, Marc Teyssier ${ }^{3,4}$, Bruno Fruchard ${ }^{4}$, \\ Jürgen Steimle ${ }^{4}$, Ana C. Baptista ${ }^{5}$, Paul Strohmeier ${ }^{4}$ \\ ${ }^{1}$ MIT Media Lab, ${ }^{2}$ Kobakant, ${ }^{3}$ Télécom Paris, \\ ${ }^{5}$ CENIMAT/I3N, NOVA University Lisbon, ${ }^{4}$ Saarland University, Saarland Informatics Campus \\ ${ }^{1}$ honnet@mit.edu, ${ }^{2}$ hannah@kobakant.at, ${ }^{5}$ anacbaptista@fct.unl.pt, ${ }^{4}\{$ lastname $\} @$ cs.uni-saarland.de
}
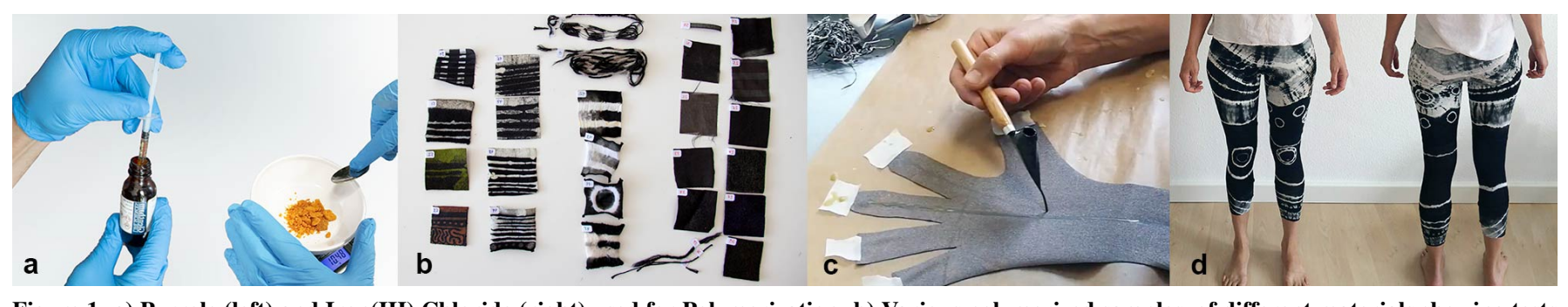

Figure 1. a) Pyrrole (left) and Iron(III) Chloride (right) used for Polymerization. b) Various polymerized samples, of different materials showing tests of batik (left) and tie-dyed traces (middle). c) Traditional manufacturing processes (here Batik) can be use to draw conductive patterns. d) Leggings augmented with polymerization to sense movement.

\section{ABSTRACT}

We present a method for enabling arbitrary textiles to sense pressure and deformation: In-situ polymerization supports integration of piezoresistive properties at the material level, preserving a textile's haptic and mechanical characteristics. We demonstrate how to enhance a wide set of fabrics and yarns using only readily available tools. To further support customisation by the designer, we present methods for patterning, as needed to create circuits and sensors, and demonstrate how to combine areas of different conductance in one material. Technical evaluation results demonstrate the performance of sensors created using our method is comparable to off-the-shelf piezoresistive textiles. As application examples, we demonstrate rapid manufacturing of on-body interfaces, tie-dyed motion-capture clothing, and zippers that act as potentiometers.

\section{Author Keywords}

eTextiles, Electro-Functionalization, In-Situ Polymerization, Piezoresistive Sensors, Wearables, Personal Fabrication

\section{CCS Concepts}

-Human-centered computing $\rightarrow$ Human computer interaction (HCI); •Hardware $\rightarrow$ Sensors and actuators;

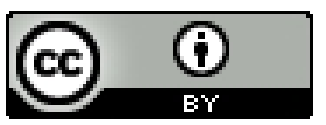

This work is licensed under a Creative Commons Attribution International 4.0 License. CHI '20, April 25-30, 2020, Honolulu, HI, USA. (C) 2020 Copyright is held by the owner/author(s). ACM ISBN 978-1-4503-6708-0/20/04.

http://dx.doi.org/10.1145/3313831.3376841

\section{INTRODUCTION}

Piezoresistive materials have become an important part of interactive eTextile systems, as they can enable fabric to sense human input, be it pressure, gestures, or motion. They are used in eTextiles for detecting body movements [10], posture [38] or gestures [29]. They are commonly used for soft pressure sensors [40] or soft pressure sensor matrices [9, 52, 31, 37], medical monitoring [20], performing arts [39] and interactive art installations [41]. Piezoresistive sensing is largely robust to the electrical noise introduced by the body [40], and piezoresistive materials are often malleable and flexible. This makes piezoresistive sensing the preferred method for wearable eTextiles, which are, by their nature, soft and conform to the shape of the body.

In this paper, we present a method of creating such piezoresistive materials, through polymerization of pyrrole (Figure 1a, left). The method presented allows conductive polymers to form in and around textiles, coating their individual fibers. The process is in-situ: polymer chains are formed in, on, and around the fabric [33], at the location where piezo-resistivity is needed.

This supports new ways of thinking about piezo-resistivity in textiles: Currently, if a sensor is to be integrated in an existing piece, the piezoresistive material must be added to the material or garment, and physically connected to conductive traces, or layered in between conductive fabric, altering the mechanical properties and haptic feel of the material or garment. Due to the in-situ nature of the polymerisation process, designers need not add external components, but can instead add piezoresistive properties to the material which they are already working with (see Figure $1 \mathrm{~b}$ and 1d). Additionally, 
this allow designers to create eTextiles independently of the availability of commercial piezoresistive fabrics with results of comparable quality to commercial solutions.

We also present methods compatible with in-situ polymerization, enabling designers to augment a fabric with patterns for general electrical functionality, that is, enable the fabric to seamlessly integrate electrical traces, resistors, sensors, heating elements, etc. However, we go beyond what is usually considered a functional material, and demonstrate how to vary the functionality within a single piece of fabric. By selectively adjusting the conductivity and piezo-resistivity of a fabric, we can seamlessly integrate basic electronic devices, such as a voltage divider, into the fabric.

The methods we present are designed to be accessible: except for the required materials, all tools necessary can be found in most kitchens. We frame polymerization as a dyeing process, and demonstrate how traditional craft methods can be used to shape the electrical properties of the outcome (see Figure 1c). With this reframing, we intend to support skilled artisans to draw on their existing expertise, and help complete novices in easily acquiring the necessary skills.

In this paper, we first present the method used for polymerizing and results of polymerizing fabrics and yarns. We then present supporting methods for fine-tuning and optimizing polymerization results before concluding with applications.

\section{RELATED WORK}

\section{eTextiles and Piezoresistive Materials}

Over the last 20 years eTextiles have developed from a fringe topic in HCI [32] to a topic of general interest. There is a growing economic relevance, highlighted by the attention eTextiles are receiving in the fashion ${ }^{1}$ and textile industries ${ }^{2}$. $\mathrm{HCI}$ research is also providing increasing attention to the topic, as visible in the increasing number of relevant publications [12]. Finally there is a vibrant and strong DIY culture around eTextiles, visible in the ease of finding high-quality instruction materials online ${ }^{3}$.

Piezoresistive materials have become an integral part of eTextile projects, as they provide an easy and robust way of sensing deformation in soft materials. However, while commonly used, the underlying mechanisms of piezo-resistivity are complex and the term is used for describing a group of related but distinct phenomena, including changes in resistivity within a material [42], as well as changes in resistivity due to changes in the quality of surface-contact [34]. Generally, one can think of piezo-resistivity as changes in the inter-molecular spacing of a material due to compression or strain, which in turn change the materials conductance (see also Figure 2). The resulting change in resistance is typically non-linear and with relatively high hysteresis $[25,26]$. The complex nature of fabric weaves makes piezoresistive sensing in textiles especially challenging, for example, the sensor-output might even be non-monotonous [27].

\footnotetext{
1e.g., https://www.fashiontech.berlin/en/

2e.g., http://wearsustain.eu/

3 e.g., http://interactex.de/, http://howtogetwhatyouwant.at/ or https://instructables.com/id/E-Textiles/
}
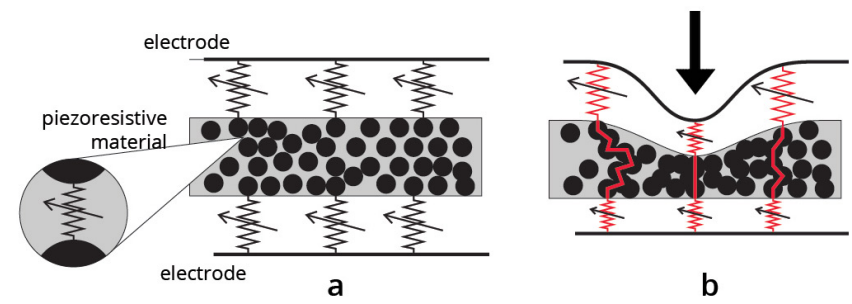

b

Figure 2. Illustration of the piezoresistive effect. When pressure is applied (right), the spacing between the conductive molecules is reduced and the surface contact is improved. Both effects create a measurable change in resistance.

Typically, piezoresistive materials are pre-fabricated as a sheet, and sandwiched between conductive electrodes for creating textile sensors [52, 31, 37, 40,38]. Other form factors include piezoresistive elastic bands [42, 41]. Conductive thread with piezoresistive coating [30] has also been demonstrated as a compact and versatile touch sensor for eTextiles. A recurring issue with these materials, however, is that they are often difficult to reproduce [30], or constrained by the availability of commercial materials such as those provided by Eeonyx $[38,9,40]$. Antistatic foil is sometimes used as alternative [15], but it is far inferior both mechanically and as a sensor. The contribution over previous work is that we provide textile designers with the ability to create high-quality piezoresistive materials themselves.

\section{Augmenting Objects with Interactive Properties}

The conventional approach for augmenting objects with sensors is to miniaturize sensing electronics and adding them to the object. Examples include inertial measurement units [36], miniaturized optical tracking systems [18], or even tape augmented with arrays of sensors [8].

An alternative approach is to coat the object to make it electrically functional. Simple sensors might be attached to the outside of objects $[17,16]$, or they might be printed on, using functional inks $[35,23]$. These surfaces need not be planar. Objectskin demonstrates how to use hydroprinting to add touch sensors and displays [14] to arbitrary geometries. Other work has explored using Electro-Impedance-Tomography [50] on spray-painted conductive surfaces, or created thin film sensing skins using mutual capacitance [51, 44]. While these approaches add sensing skins on top of objects, sensing might also be added to an object's internal structure [2].

We contribute a third way of creating interactive objects. Instead of adding components, or coating objects, with in-situ polymerization, one can change the electrical properties within the object itself.

\section{Conductive Polymers and Polymerization}

Polymerization describes the process by which monomers are combined to form a polymer chain. Here, in-situ describes that these polymer chains are created at the specific location where they are required; in, on, and around the fabric [33].

In-situ polymerization has a series of advantages over coatings: (1) the textile's mechanical properties are barely affected. As polymerization happens around and within each thread, the 
material retains the breath-ability and flexibility it had prior to polymerization. (2) as the surface area of each fibre in the material is polymerized, rather than the surface of the material, the functtionalized surface area is significantly increased [19]. (3) The mechanical bond between the fabric and the polymer is stronger, as they become integrated with each other on a fiber level.

We expand upon previous work which uses in-situ polymerization, by presenting supporting methods for selectively functionalizing fabric. To make these methods as accessible as possible, we show that traditional dying methods can be used for creating electrical components

Conductive polymers are increasingly used by the HCI community to create interactive objects. For example, Wessely deposits conductive polymers on a PDMS substrate, to sense fabric deformations [47]. PEDOT:PSS is often used as a conductor, as it can be printed with a traditional printer [24] or screen-printing [46], and can be used for creating $\mu \mathrm{m}$ thin interfaces [48]. While PEDOT:PSS has found some use within the HCI community, other conductive materials such as polypyrrole have received relatively little attention.

This is surprising, as polypyrrole is comparatively easy to polymerize. Polymerization of pyrrole has been used for creating bio-compatible batteries $[4,33]$ or electrothermal materials [49]. In fact, polymerization of pyrrole is not uncommon in the material-science communities [7].

Previous work has polymerized textiles in laboratory settings. We demonstrate that the process is simple and provide instructions which can easily be replicated by novices.

\section{THE IN-SITU POLYMERIZATION PROCESS}

Polymerization happens when a monomer is exposed to an oxidizing agent. Through oxidation, bonds to hydrogen atoms are weakened, allowing the monomer molecules to bind to each other, forming polymer chains. If other materials are present during this process - such as fabric or chemical impurities the polymer will form in and around these materials.

Organic Polymers such as polyaniline (PANI), polypyrrole (PPy) and poly (2,4-ehylenedioxythiophene) (PEDOT) have a backbone of conjugated carbon-chains. This means that they are intrinsically conductive, as electrons can move along these chains. If the polymer is doped, by, for example, adding impurities in the form of Chloride, this can add extrinsic conductivity.

We chose to explore polypyrrole as it is the easiest to synthesize in a DIY setting. Iron(III) chloride (Hexahydrate) was chose as oxidazing agent for the polymerization of pyrrole to polypyrrole. The iron(III) chloride has the additional effect of adding traces of chloride to the polymer, which increases its conductivity.

\section{Chemical Supplies}

Pyrrole solution and iron chloride were sourced from a chemical supplies dealer ${ }^{4}$. At the time of writing $25 \mathrm{~g}$ of Pyrrole

\footnotetext{
${ }^{4}$ Glentham Life Sciences Ltd
}

(approximately $75 \mathrm{ml}$ ) cost about $15 €$ and $100 \mathrm{~g}$ of iron chloride approximately $9 €$. This amount of Pyrrole can be used for polymerizing $>3 \mathrm{~m}^{2}$ cotton fabric. In comparison, $0.10 \mathrm{~m} 2$ of piezoresistive fabric by Eeonyx was sold for $25 \$$. The DIY method costs $3 \%$ of the commercial fabric, by area.

\section{Tools}

The only tools needed are bowls and a mixer for mixing the fabric and other materials, a syringe to measure the pyrrole and a scale for measuring the iron chloride. For more even results and larger batches, the mixer can be replaced with a small washing machine ${ }^{6}$. As the iron chloride water-based solution is slightly acidic, we recommend the use of glass or plastic tools.

\section{Process}

Choose amount of water - Initially one must select a good ratio of water to fabric. This is difficult to quantify, as the behavior of fabric in water can vary strongly dependent on the specific material. The goal is for the fabric to comfortably swim in the water, without lumping together, so that monomer and oxidizing agent can reach it everywhere. As a rule of thumb, by weight the ratio of water to fabric should be about $5: 1$, and by volume the amount of fabric should not exceed a water to fabric ratio of 4:3.

Dilute the monomer - Add the pyrrole, creating a water to pyrrole solution with a volume ratio of $\sim 1000: 25$. In other words, for each liter of water, add $25 \mathrm{ml}$ of pyrrole. Once the pyrrole is added, stir the solution until they are well mixed.

Add fabric - Once the fabric is added, stir gently for 10 to 15 minutes or until satisfied that the fabric has thoroughly soaked up the pyrrole mixture.

Add oxidizing agent - Add iron chloride. The mass ratio of water to iron chloride should be 100:1 (i.e., $10 \mathrm{mg}$ for every liter of water). The iron chloride might have lumped together, forming what appears as small rocks. These need to be mechanically broken down into a fine powder. Once this is done, the powder can either be directly added to the monomer mixture, or the powder can first be diluted with a small amount of water, to improve dispersion.

Wait for polymerization to complete - The mixture should be continuously stirred, so that all monomer is brought into contact with fresh iron chloride, allowing it to polymerize. Once polymerization progresses, it will become visible, as the fabric starts turning black. After about 30 minutes, when all fabric is evenly black, polymerization is complete.

Remove, Rinse and Dry - Once polymerization is complete, remove the fabric and wash it thoroughly in cold water. It can then be hung up to dry, or ironed, to speed up the drying.

\section{Factors Impacting Conductance}

While the specific results are strongly dependent on the textile chosen, there are two main mechanisms by which the conductance of the resulting functionalized textile can be manipulated, independently of the textile material.

\footnotetext{
5 https://www. adafruit.com/product/3669

6 e.g.: the DB003 by OnceConcept $(\sim 135 €)$
} 

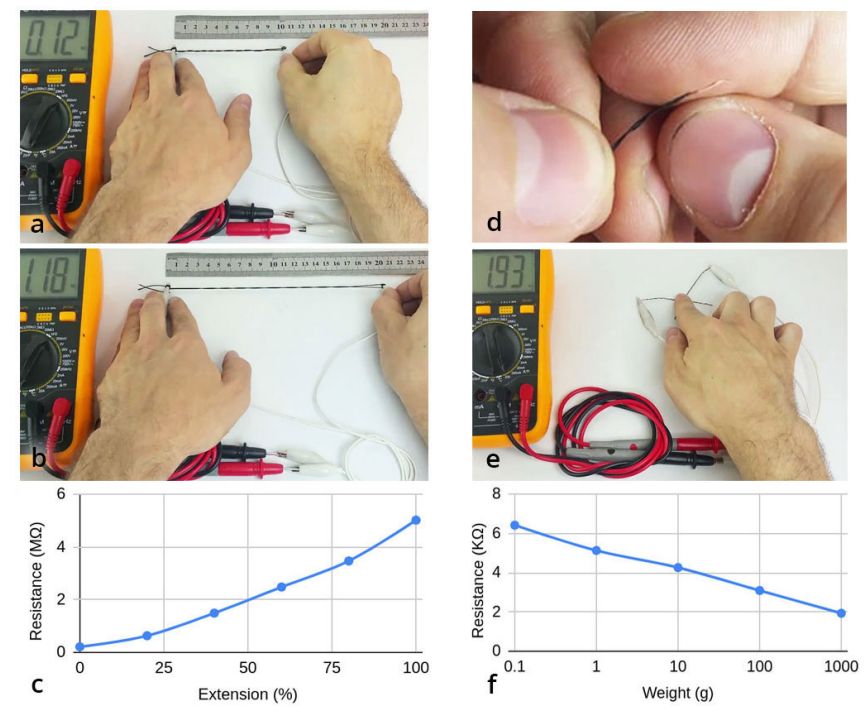

Figure 3. Stretch-sensitive yarn (left) and pressure sensitive yarn (right): Elastic piezoresistive yarns can function as stretch sensors: a) relaxed position and b) stretched position. c) elastic sensor characterization plotting resistance VS percentage stretched reveals linear behavior. d) Closeup view of a polymerized yarn with a copper conductive core that e) can sense pressure. f) pressure sensor characterization plotting resistance VS weight reveals logarithmic behavior.

The first is by changing the degree to which the fabric is polymerized. If the material is only partially polymerized, the resistance increases. Partial polymerization can be achieved by shortening the duration the fabric is submerged in the monomer bath, after the oxidation agent is added. For increased precision in stopping time, one can reduce the amount of pyrrole and iron chloride, which slows down the polymerization process.

The second is by regulating the conductivity of the polypyrrole. This is achieved by adjusting the ratio of iron chloride to pyrrole. Increasing the amount of iron chloride relative to pyrrole increases the conductivity. However, this only is effective within bounds. Above a certain level, the effect of adding more iron chloride diminishes, while adding too little iron chloride might inhibit the polymerization process (see also the formal evaluation by Baptista et al. [4]).

The conditions of polymerization can also be optimized. Distilled water can be used to make the polymerization more consistent using controlled reaction conditions, and there is evidence from the literature that polymerization at $50^{\circ} \mathrm{C}$ also has a positive effect on conductivity. To improve bonding between fabric and polymer, fabric can be pre-washed with alcohol. This is especially relevant for pre-worn fabric, as any residues of grease would inhibit polymerization.

As the results of the polymerization vary greatly based on the fabric which is polymerized, finding the optimal conditions will require some trial and error. However, once these conditions are found, the process can be repeated with consistent results (anecdotally, for two cotton textiles produced using the same methods, but from different batches, the second sample had $\sim 95 \%$ resistance of the first).

\section{PIEZORESISTIVE YARNS}

Polymerized yarns can act as stretch or pressure sensors. Functionalizing an elastic thread produced a well-performing stretch sensor (Figure $3 \mathrm{a}$ and $3 \mathrm{~b}$ ). Functionalizing cotton yarn with conductive copper core (Figure 3d), allows crossing yarns to acts as a pressure sensors (Figure $3 e$ ). We expand on the work by Parzer et al. [30] by providing an accessible implementation.

We found that the elastic yarn changes its resistance linearly with the amount it was stretched by (Figure 3c), while the resistance between two yarns with conductive core displayed a logarithmic relation to the amount of weight applied to them (Figure 3f).

\section{PIEZORESISTIVE TEXTILES}

A set of eight $5 \times 5 \mathrm{~cm}$ samples was created and compared to the non-woven fabric provided by Eeonyx. The samples were functionalized following the above instructions, but polymerized for 60 minutes. After washing and drying the samples, the transverse resistance was measured using two rigid copper electrodes of $2 \mathrm{~cm}$ diameter. For measuring the resting resistance, $10 \mathrm{~g}$ were placed on top of the top electrode, to ensure good electrode contact. As piezoresistive materials often have non-linear settling behavior, we waited for 15 seconds after the electrodes were placed before measuring the resistance. This process was repeated with $500 \mathrm{~g}$ applied, to see how the fabric is affected by pressure. The samples were stored in transparent plastic folders (non-sealed) in normal office lighting conditions. Measures were repeated after 4 months. Results of both measures are shown in Table 1.

As some samples have a very high resistance and can therefore produce a much wider resistance range, we normalize this change in resistance by dividing by the resting resistance - this produces an estimate of the sensing fidelity at the range chosen independently of the overall resistance (this is equivalent to

\begin{tabular}{lcccc}
\hline & $\mathrm{R}(10 \mathrm{~g})$ & $\mathrm{R}(500 \mathrm{~g})$ & $\mathrm{R} \Delta$ & $\mathrm{R} \Delta / \mathrm{R}$ \\
\hline Teddy Material & 120 & 19 & 101 & 0.84 \\
$80 \%$ Cotton, 20\% Polyester & 135 & 18 & 117 & 0.87 \\
Sweatshirt Fabric * & 5500 & 270 & 5230 & 0.95 \\
$96 \%$ Cotton, 4\% Elastan & 6200 & 1150 & 5050 & 0.81 \\
Paper ** & 130 & 24 & 106 & 0.82 \\
$100 \%$ Recycling Paper & 110 & 15.5 & 94.5 & 0.86 \\
Cotton (non-woven) & 88 & 16 & 72 & 0.82 \\
$100 \%$ Cotton & 81 & 12 & 69 & 0.85 \\
Lace & 75 & 8 & 67 & 0.89 \\
$95 \%$ Viscose, 5\% Elastan & 72 & 6 & 66 & 0.92 \\
Knitted Viscose & 48 & 17 & 31 & 0.65 \\
Generic Panty Hose & 35 & 4.5 & 30.5 & 0.87 \\
Nylon & 70 & 20 & 50 & 0.71 \\
Elastic Cotton * & 100 & 17 & 83 & 0.83 \\
$94 \%$ Cotton, 6\% Elastan & 2500 & 140 & 2360 & 0.94 \\
Eeonyx (non-woven) & 1950 & 200 & 1750 & 0.90 \\
& 60 & 20 & 40 & 0.67 \\
\hline
\end{tabular}

Table 1. Measures of the transverse resistance of polymerized samples. For each sample we report measures taken within two weeks of polymerization purple (top line), and 4 months after the first set of measures blue (bottom line). All results are in $\mathrm{k} \Omega$.

* These materials did not completely polymerize - see Uniformity of Polymerization in the discussion section.

** We used diaper fleece as it is made to withstand washing. 

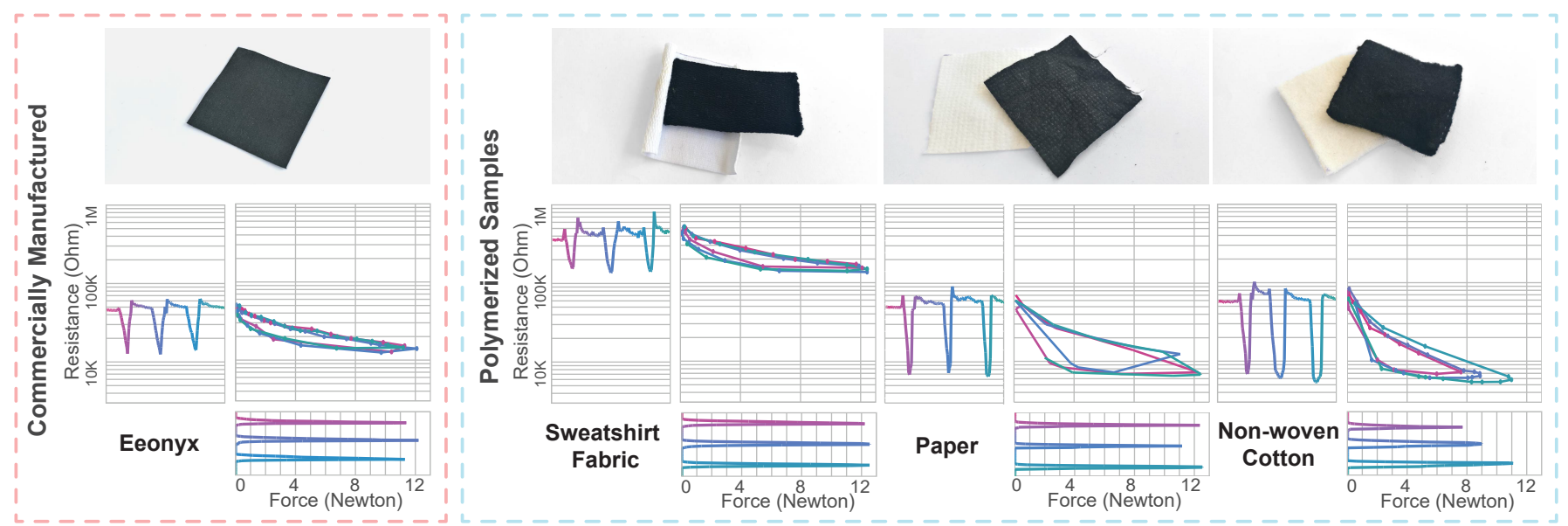

Figure 4. Comparison of Eeonyx (far left) to (from left to right) Sweatshirt Fabric, Paper (diaper fleece), and Non-Woven Cotton. Each cluster of figures shows a finger pressing the material three times, the left hand graph shows the change in resistance. The bottom graph shows the change in pressure. The top right graph shows pressure $(\mathrm{N})$ plotted against resistance $(\mathrm{R})$ to show hysteresis behavior.

the gauge factor, with a fixed force ratio, see also [25, 26]). The closer this ratio is to 1 , the better the sensor captures the range in question. This ratio should not be over-interpreted, as it does not capture hysteresis behavior or linearity of the output. So, while Table 1 appears to indicate that most samples perform better than the reference Eeeonyx material, we merely wish to point out that the samples behave similarly.

To provide a better understanding of how the materials compare, we also present measures with how they react to human pressure input. We performed three presses on each fabric and measured the resistance with a digital multimeter, and the pressure with a digital newton-metre. Again, the range of resistances of polymerized samples tended to be slightly better than the commercial sample, but the polymerize samples also tended towards higher hysteresis. In Figure 4 we plot the resistance as a function of pressure for representative samples, to show the hysteresis.

\section{PIEZORESISTIVE PATTERNS}

While large piezoresistive sheets have their use-cases - for example when creating pressure sensor matrices - typical HCI applications often require fine patterns [22]. For textiles, Piezoresistive areas should usually be constrained to the area one intends to use as a sensor. Usually this is done by cutting a smaller piece of piezoresistive material and either adhering it with fabric heat-bonding adhesive [11] or sewing it to the textile one wishes to augment. In contrast, we enable seamless integration we can control the in-situ process so polymerization only occurs where it is needed.

For ensuring accessibility we base our methods on traditional resist-based dyeing methods. Resist-based dyeing is widely used for applying colours or patterns to fabric. A substance that is impervious to the dye blocks its application to certain areas of the fabric, while other parts are exposed the dye or color. In our case, we use a material which is impervious to the oxidizing agent, and rather than using a dye, we polymerize the exposed areas. As resist-based dyeing has a rich tradition [5], learning materials are readily available. Most available information transfers directly to working with polymerization.

\section{Glue}

We found glue to be useful as a readily available resist, for quick prototyping and exploration of ideas. Hot-glue guns, for example, can make thin and accurate glue traces using paper tape as guide. The benefit of using a glue-gun is its wide availability, and that it allows for very fast and reliable prototypes. However, it can be hard to remove - the best way we found was to boil or microwave the material.

\section{Batik}

Wax can be used as a resist. A dripping tool can be formed from aluminum foil, or one can use a tjanting (a Javanese tool for applying hot wax - see Figure 1c). Patterns are drawn by hand. The wax is not affected by the polymerization process and prevents the covered material from being polymerized. It is a very tactile experience, and - similar to pen and paper drawing - allows for exploration and improvisation. Once the dyeing is complete, the wax is typically removed with boiling water or ironed out using paper towels.

The structure of the fabric, the ambient temperature, and the temperature of the wax interact strongly with how the wax flows and the resulting patterns one is able to make. For example, if either the warp or weft of a fabric are dominant, the wax tends to flow in that direction and depending on the temperature, it will penetrate further into the fabric or stay on its surface.

Figure 5a shows a sample created using batik. Here the resist was used to create two areas on the left and right of the fabric, which only connect through thin channels. Comparing measures $m 1$ and $m 3$ to $m 3$ and $m 4$ shows that the small paths successfully create areas of resistance higher by a factor of 10 .

A problem encountered using batik for polymerization is that the smallest cracks allow polymerized areas to creep into areas where one does not want them. We were able to reduce this by adding olive-oil to the wax, which makes the wax softer, and more likely to bend instead of cracking. 


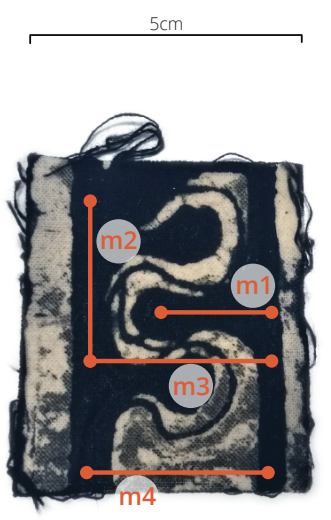

a

\begin{tabular}{c|c|c}
\multicolumn{2}{c|}{$\Omega$} & $\mathrm{cm}$ \\
\hline $\mathrm{m} 1$ & $280 \mathrm{k}$ & 2 \\
$\mathrm{~m} 2$ & $540 \mathrm{k}$ & 3 \\
$\mathrm{~m} 3$ & $2.8 \mathrm{M}$ & 3 \\
$\mathrm{~m} 4$ & $3.8 \mathrm{M}$ & 3
\end{tabular}

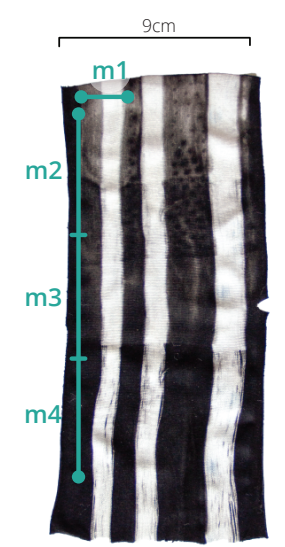

b

\begin{tabular}{c|c|c}
\multicolumn{1}{c|}{$\Omega$} & $\mathrm{cm}$ \\
\hline $\mathrm{m} 1$ & $580 \mathrm{k}$ & 2 \\
$\mathrm{~m} 2$ & $8.5 \mathrm{M}$ & 5 \\
$\mathrm{~m} 3$ & $20 \mathrm{M}$ & 5 \\
$\mathrm{~m} 4$ & $40 \mathrm{M}$ & 5
\end{tabular}
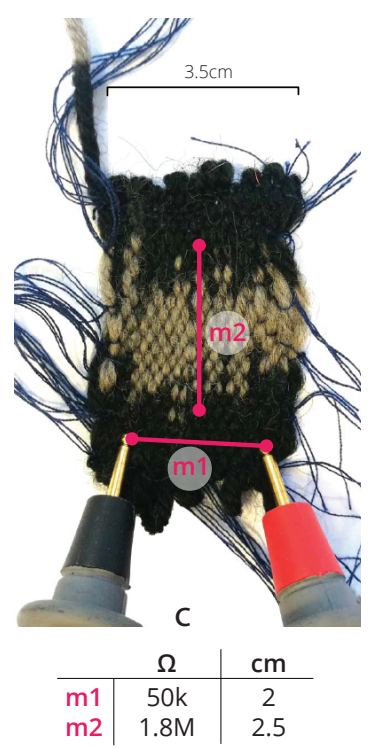

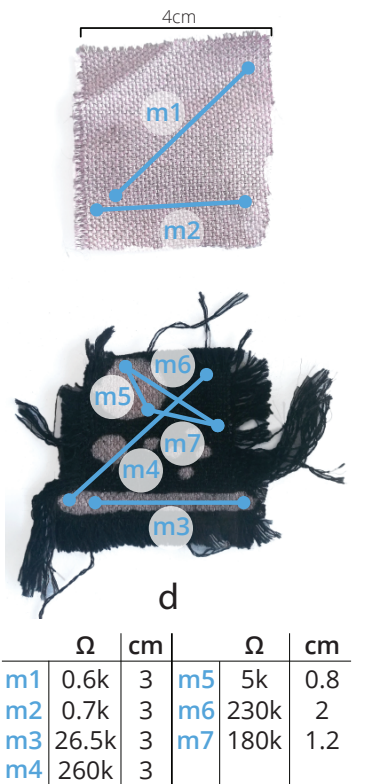

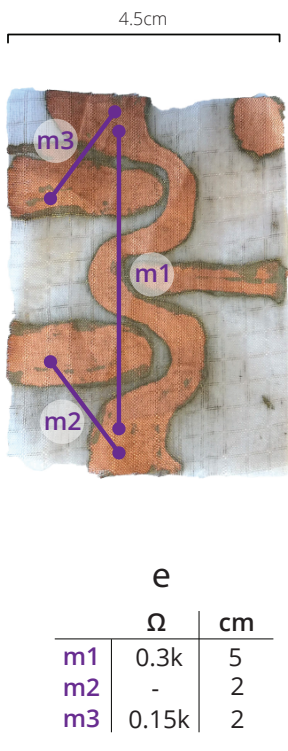

Figure 5. Measures of resistance values over several patterned fabrics: (a) Calico (batik), (b) Cotton (tie-dyed), (c) Wool (woven), (d) Bamboo/Silver (polymerized using batik on conductive fabric), (e) Nylon/Copper (etched using batik).

\section{Tie-Dye}

The method consists of folding, twisting or applying pressure on the fabric with strings or rubber bands. The selective application of pressure determines if the fabric can absorb the dye, or in our case whether it is polymerized or not.

Tie dying introduces an additional layer of control. Working with folds allows the creation of repeating patterns and embedding objects provides the ability to add specific predefined shapes. By applying a gradient of pressure to the tied object, one can also introduce gradients of resistance into the polymerized areas. For example, the sample shown in Figure 5b was created by wrapping fabric around a plastic tube. The white areas were tied down with thread, so they are not polymerized. The black areas were exposed, however, as it was wrapped, the top end is covered by two layers of textiles and the middle is covered by one layer. Consequently the resistance towards the top is highest, while it increases towards the bottom, as can be seen comparing measures $m 2, m 3$, and $m 4$. Measure $m 1$ shows that the tied-down areas successfully act as insulators, there is barely any conductivity between the stripes.

Compared to traditional batik, tie-dyeing is better for larger areas, as creating large areas of resist is less laborious. However, tie-dying has its own issue with scale, as one needs to ensure that the pyrrole and the oxidizing agent can reach all required areas. This can be a problem if the fabric is too thick or if too many layers of fabric are stacked.

\section{Making Patterns from Yarn}

Resist-based selective polymerizing need not be applied to finished textiles. Instead it can also be used for creating a yarn which is piezoresistive in selective areas. Such selectively polymerized threads can later be woven or knitted to create custom shapes and patterns.
As has been demonstrated in previous work, resistive yarn can be added to traditional weaves. However, these yarns need not be restricted to discrete rows and columns. Using patterned yarn, resistive or non resistive areas can be created at will. The woven sample (Figure 5c) shows that the conductance between pre-polymerized yarns that touch is strong enough to form continuous piezoresistive areas. This process, again, is borrowed from traditional textile dying technique and is commonly referred to as ikat.

Combined with the advance of custom thread-spinning technologies ${ }^{7}$ which allow dynamic adjusting of thickness and material composition of a yarn, ikat has the potential for enabling the creation of fully customizable functional fabrics.

\section{Using Rapid Fabrication Tools}

While traditional hand-craft can provide strong results, we were also interested in creating methods which allowed for a higher level of algorithmic control. Here our best results were based around laser-cut stencils.

We explored two strategies. The first is to create a laser-cut negative and pressure fitting the laser-cut pattern to the front

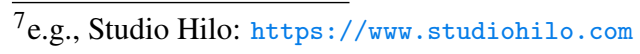
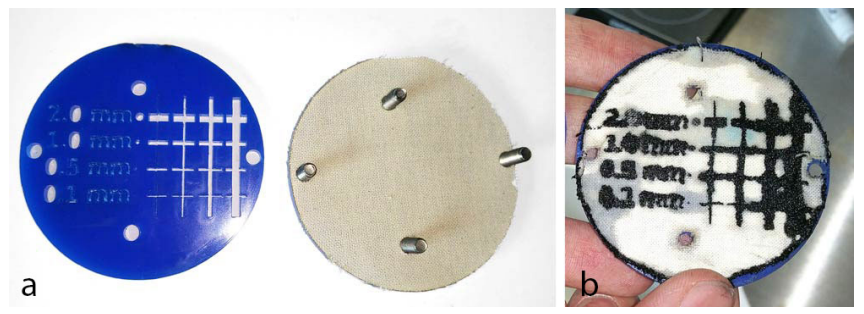

Figure 6. A laser cut stencil (a-left) pressed against a fabric (a-right) can be used to perform selective polymerization (b). 
and back of the fabric, fastening it with screws or clamps (Figure 6a). We then polymerize the entire rigid object (Figure $6 b)$. For larger textiles this might create a rigid object which cannot practically be fit into a mixing container. In those cases we instead create a positive image of the desired shape, and again pressure fit it to the front and back of a fabric. We then fill the exposed negative shape with wax. The wax then acts as a resist during polymerization (as shown in Video Figure). After polymerization, the wax can be ironed out.

Alternatively one can also 3D print a single layer shape directly on to the textile. If the textile is strong enough, the printed layer can be pulled off after polymerization. Boiling the fabric weakens the bond between the 3D printed layer.

\section{INTEGRATING HIGHLY CONDUCTIVE FABRIC}

While custom piezoresistive patterns already provide interesting opportunities, seamless integration is further supported by embedding conductors into the fabric. To achieve this level of integration, we use commercially available conductive fabric ${ }^{8}$. In this section we discuss how conductive materials can be used in combination with polymerization.

\section{Polymerizing Conductive Fabrics}

Conductive fabric typically consists of a synthetic fibers which are coated in a thin conductive layer. When polymerizing conductive fabric, the oxidizing agent oxidizes the conductive layer, removing the conductivity of the fabric. After the polymerization process, the fabrics electrical properties are no longer distinguishable from a polymerized non-conductive sample.

Coating part of a sample with a resist before polymerization allows the combination of highly conductive and piezoresistive areas. For most fabric we tested (successful samples included: SafetySilk, Soft\&Safe, Silverell sold by lessEMF as well as Techniktex p130 and Shieldex Balingen sold by Statex) the polymerized areas connected to the conductive areas. This allows integrating an area that acts as a sensor within highconductive material, which can act as electrical connection to a microcontroller or other parts of the circuitry.

This is demonstrated in Figure 5d. The top shows an unmodified sample, the bottom shows a sample where a resist was applied using batik. The diagonal measures $(\mathrm{ml}$ and $\mathrm{m} 4)$ and the horizontal measures ( $m 2$ and $m 3$ ) show how the behavior of the fabric is changed after polymerization. Measure $m 6$ shows a measure that spans a piezoresistive and a conductive zone. Measures $m 5$ and $m 7$ show measures going up to the border of the two areas.

We found that wax-based resists worked best for this type of process. Tie-dyed samples often had areas of poor conductance between conductive and piezoresistive areas.

\section{Etching Conductive Fabrics}

For successfully making a circuit, one must not only provide paths through which signals can propagate with minimal re-

\footnotetext{
${ }^{8}$ While we would prefer to create these ourselves too, sourcing conductive fabric is much simpler than piezoresistive fabric. We obtain many of our fabrics from Statex Produktions- und Vertriebs GmbH.
}

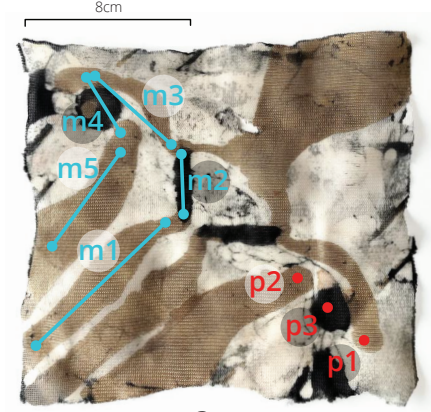

a

\begin{tabular}{|c|c|c|c|c|c|}
\hline \multicolumn{2}{|r|}{$\Omega$} & $\mathrm{cm}$ & \multicolumn{2}{|r|}{$\Omega$} & $\mathrm{cm}$ \\
\hline $\mathrm{m}^{-}$ & $800 \mathrm{k}$ & 7 & $\mathrm{~m} 3$ & $5 \mathrm{k}$ & 4.5 \\
\hline$m_{\text {laxed }}^{2}$ & $100 \mathrm{M}$ & 2.5 & m4 & - & 3 \\
\hline$\underset{\text { tretch) }}{\mathrm{m} 2}$ & $20 \mathrm{M}$ & 4 & m5 & $200 \mathrm{k}$ & 6 \\
\hline
\end{tabular}

Figure 7. Hybrid textile, combining etching and polymerization to create a complex electronic circuit. Black areas are piezo resistive, brown areas are conductive, while white areas are non-conductive. The textile has two stretch sensors (centre, elongated black strips) and two pressure sensors integrated. The pressure sensor is used by folding the textile.

sistance - one also needs insulators. When working with conductive textiles, we must create such non-conductive areas.

A simple etching process can be used for creating nonconductive areas in conductive fabric. After applying a wax resist, the fabric is placed in a mixture of vinegar $(24 \%)$ and Hydrogen Peroxide (30\%) mixed in a ratio of 2:1. After about 5 minutes one can see first visible changes in the fabric and after about 15 minutes the fabric usually is fully etched, however the duration is dependent on the amount of metal which needs to be removed: As more metal is dissolved, the potency of the mixture is reduced, and etching gradually slows down. Once removed, the fabric needs to be thoroughly washed, as otherwise the edges of the conductive areas are further oxydized forming a barrier of low conductivity on their edge.

In Figure 5e we present such an etched sample. Again, the resist was applied using batik. We see that the copper preserved its high conductance $m l$ and that the bottom left pad $m 2$ is not electrically connected to the other conductive areas. However, we see high conductivity between the top left pad $m 3$ and other areas. This suggests that the sample was removed from the etching bath prematurely.

\section{Combining Etching and Polymerizing}

Once the desired pattern of conductive and insulating areas is created, piezoresistive sensors can be integrated in the circuit using polymerization. Again, a wax resist needs to be applied to all areas which should not be polymerized. The surface to be polymerized can be either etched, or left conductive.

A sample created using Shieldex Balingen can be seen in Figure 7a. The brown areas are conductive, the black areas piezoresistive and the white areas act as insulator. The textile contains two stretch sensors (elongated black areas in center) and two pressure sensors (round black areas towards top left and bottom right). The top right conductor might be connected to a GND, and the bottom left conductors to analog inputs 
with activated pull up resistors. Alternatively - if for example, only using the two central stretch sensors - the top right corner might be connected to an analog input, and the other side of each stretch sensor connected to GND and V+ respectively. They would then act as a voltage divider. Figure 7 provides more information as to the samples electrical characteristics.

The pressure sensor is designed to be used when the fabric is folded as seen in Figure 7b. By folding the fabric twice, conductor $p l$ connects at the top of the piezoresistive element $p 3$ and the conductor $p 2$ connects from below. Measuring from point $p 1$ to point $p 2$ while pressing the fabric provides a range of resistance from $50 \mathrm{k} \Omega$ to $6 \mathrm{M} \Omega$. This layering approach was implemented for measuring changes in pressure perpendicularly to the material, as for example done by zPatch [40]. However, we found that it was not always necessary and that often pressure can be measured in plane with the fabric: If two conductors are separated by an area of piezoresistive fabric, then compressing this fabric decreases the resistance between the conductors.

\section{APPLICATION EXAMPLES}

We present a series of objects which take advantage of the in-situ nature of the polymerization process. Instead of mechanically adding sensor layers to these objects, we functionalized them, starting from existing objects and adding sensing abilities without introducing mechanical changes or impeding their original purpose.

\section{Tie-Dyed Motion Capture}

Piezoresistive materials are often used for capturing human motion. For example, Parzer et al. presented a sleeve, consisting of piezoresistive fabric sandwiched between conductive fabric, that can measure elbow movements [31] and Strohmeier et al. integrated piezoresistive polymer bands into a wristwarmer to measure movement of the wrist [42]. PolySense allows the creation of similar garments, but with significantly reduced mechanical complexity. We demonstrate this by augmenting a pair of (1) cotton librarian gloves (women's medium, roughly $185 \mathrm{~mm}$ by $195 \mathrm{~mm}$ textile, <50 g) and a pair of self made (2) cotton leggings (roughly $660 \mathrm{~mm}$ by $800 \mathrm{~mm}, 150 \mathrm{~g}$ ) with tie-dyed polymerized patterns to make them sensitive to motion. The fabric was polymerized for 40 minutes, according to the instructions provided in this paper.

(1) the glove was used to control a kinematic model of a hand (Figures $8 \mathrm{a}$ and $8 \mathrm{~b}$ ). To make it, the cotton glove was polymerized with tight strings at various phalanx levels to isolate them. The resistance of pressure sensitive areas on the knuckles were mapped to joints of the model, using forward kinematics. The software and firmware of this example are available on GitHub ${ }^{9}$.

(2) the leggings were used to measured knee flex movements (Figures 1d). These were finished to a fully integrated device. The resistance at the knees was measured using a conductive undergarment. The values were sent to a computer using the WiFi capabilities of the x-OSC board. Technical details

\footnotetext{
${ }^{9}$ Augmented glove documentation:

https://github.com/counterchemists/glove_viz
}
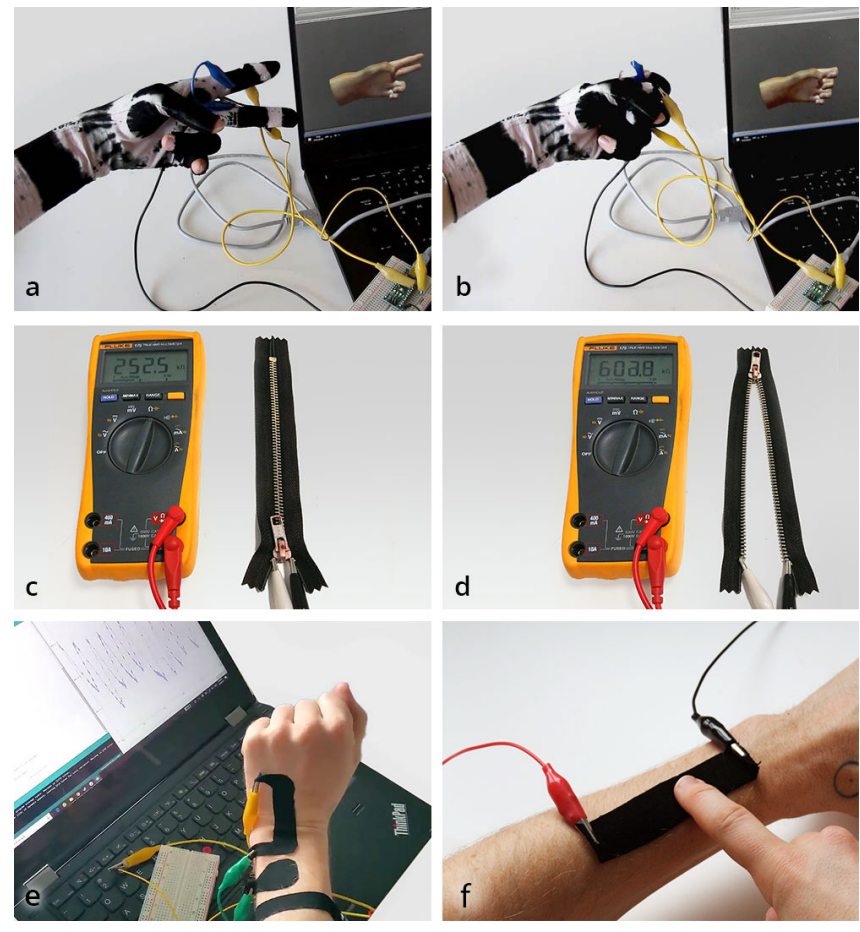

Figure 8. Application examples of polymerization. Cotton gloves capture hand movements ( $a$ and $b$ ), a zipper is turned into a linear potentiometer ( $c$ and $d$ ), and kinesio tape becomes a tool for rapidly prototyping on-skin UI's.

of this project are documented on GitHub ${ }^{10}$. The motioncapturing leggings were used to control the slide transitions at a presentation attended by $\sim 200$ people, during the summer edition of CCC in 2019.

\section{Augmenting Mechanical Objects}

When everyday objects are augmented with electrical functions, for example, as demonstrated by Gröger et al. [14] their physical characteristics provide unique opportunities. For example, Weigel et al. [46] and Bergström et al. [6] show how features of the body, such as wrinkles, knuckles or moles can be used for interaction. We show that with increasing complexity of the polymerized object, surprising results can be achieved.

We polymerized zippers (approximately $120 \mathrm{~mm}$ by $30 \mathrm{~mm}$ ), made of cotton, with metal teeth. We polymerized longer than usual ( $\sim 60$ minutes) to ensure electrical contact between polymerized cotton and metal teeth. In some cases, the polymerization slightly oxidizes the metal teeth, degrading electrical contact between them. The oxidized layer can be removed using flux and a hot air gun. The oxidation problem can be avoided by using stainless steel zippers.

The resulting zipper can be used similar to a linear potentiometer: The resistance measured from the left side of the zipper to the right side of the zipper is highest when it is fully zipped (Figure 8c), as the electricity can flow through the metal teeth and lowest when it is unzipped (Figure 8d), as the

\footnotetext{
${ }^{10}$ Augmented leggings documentation:

https://github.com/counterchemists/leggings
} 
electricity needs to travel through the entire fabric. We created multiple of these zipentiometers, and found resistance values from $\sim 200 \mathrm{k} \Omega$ to $\sim 600 \mathrm{k} \Omega$ when the zipper was closed and $\sim 500 \mathrm{k} \Omega$ to $\sim 4 \mathrm{M} \Omega$ when the zipper was unzipped.

\section{On-Body Uls using Kinesio Tape}

The ability to augment mechanically complex objects can be used for simplifying rapid prototyping. For example, Onbody interfaces and epidermal devices are receiving increasing attention within HCI (e.g.: [45, 21, 28]), but are typically non-trivial to prototype. We show that polymerizing tape can provide a fast way for rapidly creating functional prototypes of on-body interfaces:

Kinesio tape is a readily available soft sticky tape designed to comply to and attach to the skin. It consists of a glue layer attached to a woven fabric. By polymerizing kinesio tapes, we create a simple substrate for creating custom onbody interfaces. The polymerization process functionalizes the fibers of the woven fabric, while not affecting the glue layer. This creates a versatile bio-compatible sensor, that only needs to be cut in the desired shape and applied directly to the body, including (1) motion sensors, (2) touch sensors and (3) position sensors.

(1) Motion Sensor: A strip of $\sim 300 \mathrm{~mm}$ by $50 \mathrm{~mm}$ was polymerized according to the instructions provided in this paper. If a part of this strip is placed on a body joint, the resistance changes as the tape is stretched or compressed by motion (Figure $8 \mathrm{e}$ - the example used in the Video is documented on GitHub $^{11}$.

(2) Touch Sensor: By placing grounded pads on a body part, and connecting a finger to a microcontroller input with a pullup resistor, one can detect touch. Indeed, when the finger touches the grounded pad, the input is pulled low. Multiple touch-sensors can be used by pulling the pads low sequentially, and disconnecting them the rest of the time by setting pins to high impedance.

(3) Position sensor: Finally, a linear touch sensor can be made by cutting a strip of tape, and connecting one side of it to GND (Figure 8f, black cable) and the other to V+ (red cable). If the finger touching it is connected to an analog input, the voltage measured at the finger will be highest when the finger is close to V+ and lowest when it is close to GND. In the case of Figure $8 \mathrm{f}$, the finger is about one third of the way towards V+ from GND - the voltage measured at the finger will therefore also be about on third of $\mathrm{V}+$.

\section{DISCUSSION}

This project had a very simple origin: we wanted to find a reliable source for creating the piezoresistive central layer for a pressure sensor matrix. After speaking with manufacturers, we understood that we depended on specialized companies that produce a few samples made of specific textiles. As we could not produce such a material ourselves, we were forced to adapt our designs to what was available - we never had the opportunity to experiment with modifying the properties of

\footnotetext{
${ }^{11}$ Wrist sensor documentation:

https://github.com/counterchemists/perlin/
}

the piezoresistive material ourselves. Treating the piezoresistive textiles as something immutable prevents us from fully exploring the design space. These considerations made it clear that we need an accessible process, which not only makes it possible to copy and reproduce technologies based on piezoresistivity, but also provides a platform for playful exploration. The example applications presented in this paper demonstrate that we achieved this goal.

\section{Sensor Behavior}

All piezoresistive fabric produced using polymerization displayed pressure sensitive behavior. However, not all samples were sensitive to stretching. We assume this is caused by the structure of the textiles: For the polymerized stretch thread (See Figure 3, left), the fibers actually stretch, when the thread is stretched. With many fabrics, this is not the case. For example, in Figure 9, bottom, we see the same textile stretched (Figure 9d) and relaxed (Figure 9c). Most of the strain is absorbed by the structure of the fabric. Rather than extending individual fibers, the change in area is caused by a restructuring of individual fibers. As the fibers themselves are not stretched, this does not cause a change in resistance. While this is not the case for all textiles, it might be considered a drawback of polymerization - if the fabric were coated, it would demonstrate a change in resistance.

In practice this was a non-issue, as - if the fabric is stretched over a joint - pressure is also exerted on the fabric, which leads to a change in resistance. In fact, it might even provide a higher level of control over fabrics which are effected both by extension and pressure.

\section{Advanced Materials}

Paying detailed attention to the specific structure and composition of a fabric does not only help understanding its sensing properties, but might also be key for creating even more advanced fabric circuitry. If one polymerizes a hybrid fabric consisting of more than one material, the polymer does not interact with the fabric as a whole, but rather with its individual fibers, which reacts differently to the polymerization process. Figure 9a and $9 \mathrm{~b}$ shows hybrid textiles consisting of fibers which were polymerized (black) and fibers which were not polymerized (white/yellow). A way of quantifying how well a fiber will polymerize might be by the degree it is able to soak up fluids [1].

Considering the micro-structure of polymerizing fabric might open up new opportunities, such as the design of sensors which require combining materials with varying electrical properties, such as dielectrics, conductors and semi-conductors. One could implement piezoresistive traces in the $\mu \mathrm{m}$ range, or designs which combine different electrical properties, such as dielectrics, conductors and semi-conductors on a microscale. This might enable new sensing approaches, for example, measuring interstitial capacitance between fibers, or other mems-inspired designs.

\section{Scaling}

Polymerization can be used both for making fine-detailed functionalization, as well as functionalizing large batches of material. As described, we can polymerize single fibers: a 


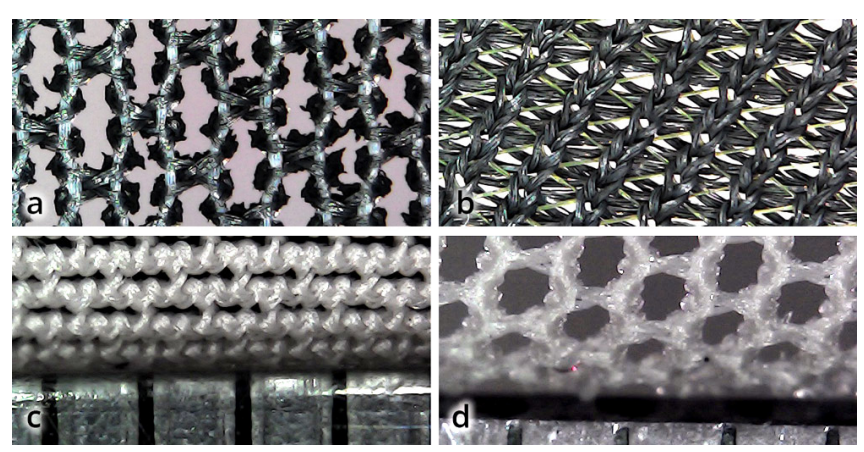

Figure 9. Various textiles with 1000x magnification. Black fibers are polymerized, white ones are not. a) Powermesh (82\% Nylon and $18 \%$ Spandex), b) Techniktex p130 (78\% Polyamide and $18 \%$ Elastomer). c) Powermesh in relaxed state (rotated 90 degrees) and d) Powermesh when stretched. Below a scale with $\mathrm{mm}$ spacing is visible.

single filament of Nylon integrated in a spandex fabric, would create a trace in the 10-30 $\mu \mathrm{m}$ range. The largest fabric we have polymerized are the leggings shown in Figure 1d, and the smallest feature we created was $\sim 0.8 \mathrm{~mm}$ wide, using the stencil shown in Figure 6. Using the setup described in this paper, we could theoretically polymerize $15 \mathrm{~m}^{2}$ of cotton jersey (assuming $150 \mathrm{~g}_{\text {per }} \mathrm{m}^{2}$ ).

\section{Uniformity of Polymerization}

As reported in the piezo resistive textiles section, not all fabric always polymerized completely. In that specific case, the polymerization was impeded because the samples were woven in a structure which makes them roll up when cut. We report the results anyway, to highlight the wide range of effects which can be achieved (see also Table 1).

Uniformity of polymerization is heavily dependent on how well the oxidizing agent reaches all areas of the textile. If the textile is clumped together, or if the oxidizing agent does not disperse, the fabric will be non-uniform. These problems are largely avoided when a washing machine is used: We measured the transverse resistance over the area of an A4 sized sheet of polymerized Lycra, using $3 \mathrm{~cm}$ grid spacings. We calculated the average absolute deviation from the mean resistance to be $<7 \%$.

\section{Limitations}

While the samples created by us did not show obvious signs of deterioration, polymerized textiles should be handled with care. For example, exposure to water in general, and specifically machine washing polymerized textiles should be avoided. There are also complex interactions between high temperature, humidity and oxygen exposure, which together also have an adverse effect on longevity of the fabric. A detailed investigation of this is presented by Banaszczyk et al. [3].

Polypyrrole is also known for its vulnerability to UV light [13]. To assess if this impacts the polymerized Lycra, we placed a sample of polymerized Lycra in a 36 Watt UV chamber $(254 \mathrm{~nm})$. We measured conductivity before exposure and after $1,2 \& 3$ hours. The measured conductivity decreased by $25 \%$ after initial exposure to UV, and remained stable.
Having pointed out these limitations, we wish to stress that in general, polypyrrole is considered to have "good stability in ambient conditions" [13] - which we could confirm: as highlighted in Table 1, on average the resistance of samples stayed stable over a four month period.

Most of the methods presented in the paper rely on manual traditional methods, which might lack precision in demanding scenarios. Thin materials could in the future be applied with resist using a solid-ink printer, similar to those used for DIY fabrication of flexible circuits [43]. However, we do not believe that this would work well for thicker materials. Commercial wax $3 \mathrm{D}$ printers ${ }^{12}$ may provide inspiration for a more consistent process. Looking even further towards the future, custom weaving or knitting devices coupled with custom threads with variable properties will provide even more control on the material level.

\section{CONCLUSION}

PolySense is an accessible method for enhancing textiles and other fibrous materials with custom piezoresistive properties. We have shown that in-situ polymerization enables seamless integration at the material level, preserving the textiles mechanical properties and using only readily available tools. To further support customisation by the designer, we presented methods for patterning, as needed to create circuits and sensors, and demonstrated how to combine areas of varying conductivity in one material. The performance of sensors created using our method are comparable to off-the-shelf piezoresistive textiles.

We are sharing this method, to support the creation of exciting new interactive prototypes. We are looking forward to learning what PolySense will enable others to do.

\section{ACKNOWLEDGEMENTS}

This project received funding from the European Research Council (ERC StG Interactive Skin 714797). This work would not exist without Joana Neto, thank you. We are also grateful for the support and feedback from Yvonne Jansen and Gilles Bailly. Finally, we would like to thank our DataPaulette colleagues for their inspiring enthusiasm.

\section{REFERENCES}

[1] Linden Allison, Steven Hoxie, and Trisha Andrew. 2017. Towards Seamlessly-Integrated Textile Electronics: Methods to Coat Fabrics and Fibers with Conducting Polymers for Electronic Applications. Chem. Commun. 53 (05 2017). DOI :

http://dx.doi.org/10.1039/C7CC02592K

[2] Moritz Bächer, Benjamin Hepp, Fabrizio Pece, Paul G. Kry, Bernd Bickel, Bernhard Thomaszewski, and Otmar Hilliges. 2016. DefSense: Computational Design of Customized Deformable Input Devices. In Proceedings of the 2016 CHI Conference on Human Factors in Computing Systems (CHI '16). ACM, New York, NY, USA, 3806-3816. DOI : http://dx.doi.org/10.1145/2858036.2858354

\footnotetext{
${ }^{12}$ Such a technology is used by https: //www. solidscape.com/
} 
[3] Jędrzej Banaszczyk, Andrzej Rybak, and Mateusz Odziomek. 2015. Aging of Polypyrrole Coated Fabrics Potted in Epoxy. Fibres and Textiles in Eastern Europe 23 (03 2015), 79-83.

[4] A. C. Baptista, I. Ropio, B. Romba, J. P. Nobre, C. Henriques, J. C. Silva, J. I. Martins, J. P. Borges, and I. Ferreira. 2018. Cellulose-based electrospun fibers functionalized with polypyrrole and polyaniline for fully organic batteries. Journal of Materials Chemistry A 6, 1 (dec 2018), 256-265. DOI : http://dx.doi.org/10.1039/C7TA06457H

[5] N. Belfer. 1992. Batik and Tie Dye Techniques. Dover Publications.

[6] Joanna Bergstrom-Lehtovirta, Sebastian Boring, and Kasper Hornbundefinedk. 2017. Placing and Recalling Virtual Items on the Skin. In Proceedings of the 2017 CHI Conference on Human Factors in Computing Systems (CHI '17). Association for Computing Machinery, New York, NY, USA, 1497-1507. DOI : http://dx.doi.org/10.1145/3025453.3026030

[7] Lina M Castano and Alison B Flatau. 2014. Smart fabric sensors and e-textile technologies: a review. Smart Materials and Structures 23, 5 (2014), 053001. DOI : http://dx.doi.org/10.1088/0964-1726/23/5/053001

[8] Artem Dementyev, Hsin-Liu (Cindy) Kao, and Joseph A. Paradiso. 2015. SensorTape: Modular and Programmable 3D-Aware Dense Sensor Network on a Tape. In Proceedings of the 28th Annual ACM Symposium on User Interface Software \& Technology (UIST '15). Association for Computing Machinery, New York, NY, USA. DOI : http://dx.doi.org/10.1145/2807442.2807507

[9] Maurin Donneaud, Cedric Honnet, and Paul Strohmeier. 2017. Designing a Multi-Touch eTextile for Music Performances. In Proceedings of the International Conference on New Interfaces for Musical Expression. 7-12. http://www.nime . org/proceedings/2017/nime2017

[10] Yu Enokibori and Kenji Mase. 2014. Human Joint Angle Estimation with an e-Textile Sensor. In Proceedings of the 2014 ACM International Symposium on Wearable Computers (ISWC '14). ACM, New York, NY, USA, 129-130. DOI :

http://dx.doi.org/10.1145/2634317.2634331

[11] Rachel Freire, Cedric Honnet, and Paul Strohmeier. 2017. Second Skin: An Exploration of eTextile Stretch Circuits on the Body. In Proceedings of the Eleventh International Conference on Tangible, Embedded, and Embodied Interaction (TEI '17). ACM, New York, NY, USA, 653-658. DOI :

http://dx.doi.org/10.1145/3024969.3025054

[12] Rachel Freire, Paul Strohmeier, Cedric Honnet, Jarrod Knibbe, and Sophia Brueckner. 2018. Designing eTextiles for the Body: Shape, Volume \&\#38; Motion. In Proceedings of the Twelfth International Conference on Tangible, Embedded, and Embodied Interaction (TEI '18). ACM, New York, NY, USA, 728-731. DOI : http://dx.doi.org/10.1145/3173225.3173331
[13] Pavel Galár, Branislav Dzurnak, Petr Maly, Jan Čermák, Alexander Kromka, Mária Omastová, and Bohuslav Rezek. 2013. Chemical Changes and Photoluminescence Properties of UV Modified Polypyrrole. International journal of electrochemical science 8 (01 2013), 57-70.

[14] Daniel Groeger and Jürgen Steimle. 2018. ObjectSkin: augmenting everyday objects with hydroprinted touch sensors and displays. Proceedings of the ACM on Interactive, Mobile, Wearable and Ubiquitous Technologies 1, 4 (2018), 134.

[15] Florian Heller, Stefan Ivanov, Chat Wacharamanotham, and Jan Borchers. 2014. FabriTouch: Exploring Flexible Touch Input on Textiles. In Proceedings of the 2014 ACM International Symposium on Wearable Computers (ISWC '14). ACM, New York, NY, USA, 59-62. DOI : http://dx.doi.org/10.1145/2634317.2634345

[16] David Holman, Nicholas Fellion, and Roel Vertegaal. 2014. Sensing Touch Using Resistive Graphs. In Proceedings of the 2014 Conference on Designing Interactive Systems (DIS '14). Association for Computing Machinery, New York, NY, USA, 195-198. DOI : http://dx.doi.org/10.1145/2598510.2598552

[17] David Holman and Roel Vertegaal. 2011. TactileTape: Low-Cost Touch Sensing on Curved Surfaces. In Proceedings of the 24th Annual ACM Symposium Adjunct on User Interface Software and Technology (UIST' '11 Adjunct). Association for Computing Machinery, New York, NY, USA, 17-18. DOI : http://dx.doi.org/10.1145/2046396.2046406

[18] Cédric Honnet and Gonçalo Lopes. 2019. HiveTracker: 3D Positioning for Ubiquitous Embedded Systems. In Adjunct Proceedings of the 2019 ACM International Joint Conference on Pervasive and Ubiquitous Computing and Proceedings of the 2019 ACM International Symposium on Wearable Computers (UbiComp/ISWC '19 Adjunct). ACM, New York, NY, USA, 288-291. DOI :

http://dx.doi.org/10.1145/3341162.3349295

[19] Bingxiang Huang, G.J. Kang, and Y. Ni. 2006. Preparation of conductive paper by in-situ polymerization of pyrrole in a pulp fibre system. Pulp and Paper Canada 107 (02 2006), 38-42.

[20] JW Jeong, YW Jang, I Lee, S Shin, and S Kim. 2009. Wearable respiratory rate monitoring using piezo-resistive fabric sensor. In World Congress on Medical Physics and Biomedical Engineering, September 7-12, 2009, Munich, Germany. Springer, 282-284.

[21] Hsin-Liu Cindy Kao, Abdelkareem Bedri, and Kent Lyons. 2018. SkinWire: Fabricating a Self-Contained On-Skin PCB for the Hand. Proc. ACM Interact. Mob. Wearable Ubiquitous Technol. 2, 3, Article Article 116 (Sept. 2018), 23 pages. DOI : http://dx.doi.org/10.1145/3264926 
[22] Kunihiro Kato and Homei Miyashita. 2015. ExtensionSticker: A Proposal for a Striped Pattern Sticker to Extend Touch Interfaces and Its Assessment. In Proceedings of the 33rd Annual ACM Conference on Human Factors in Computing Systems (CHI'15). Association for Computing Machinery, New York, NY, USA, 1851-1854. DOI :

http://dx.doi.org/10.1145/2702123.2702500

[23] Yoshihiro Kawahara, Steve Hodges, Benjamin S Cook, Cheng Zhang, and Gregory D Abowd. 2013. Instant inkjet circuits: lab-based inkjet printing to support rapid prototyping of UbiComp devices. In Proceedings of the 2013 ACM international joint conference on Pervasive and ubiquitous computing. ACM, 363-372.

[24] Arshad Khan, Joan Sol Roo, Tobias Kraus, and Jürgen Steimle. 2019. Soft Inkjet Circuits: Rapid Multi-Material Fabrication of Soft Circuits Using a Commodity Inkjet Printer. In Proceedings of the 32nd Annual ACM Symposium on User Interface Software and Technology (UIST '19). Association for Computing Machinery, New York, NY, USA, 341-354. DOI : http://dx.doi.org/10.1145/3332165.3347892

[25] An Liang, Rebecca Stewart, and Nick Bryan-Kinns. 2019a. Analysis of Sensitivity, Linearity, Hysteresis, Responsiveness, and Fatigue of Textile Knit Stretch Sensors. Sensors 19, 16 (2019), 3618.

[26] An Liang, Rebecca Stewart, Rachel Freire, and Nick Bryan-Kinns. 2019b. Effect of Bonding and Washing on Electronic Textile Stretch Sensor Properties. In Adjunct Proceedings of the 2019 ACM International Joint Conference on Pervasive and Ubiquitous Computing and Proceedings of the 2019 ACM International Symposium on Wearable Computers (UbiComp/ISWC '19 Adjunct). ACM, New York, NY, USA, 121-124. DOI : http://dx.doi.org/10.1145/3341162.3343817

[27] Ruibo Liu, Qijia Shao, Siqi Wang, Christina Ru, Devin Balkcom, and Xia Zhou. 2019. Reconstructing Human Joint Motion with Computational Fabrics. Proc. ACM Interact. Mob. Wearable Ubiquitous Technol. 3, 1, Article 19 (March 2019), 26 pages. DOI : http://dx.doi.org/10.1145/3314406

[28] Eric Markvicka, Guanyun Wang, Yi-Chin Lee, Gierad Laput, Carmel Majidi, and Lining Yao. 2019. ElectroDermis: Fully Untethered, Stretchable, and Highly-Customizable Electronic Bandages. In Proceedings of the 2019 CHI Conference on Human Factors in Computing Systems (CHI '19). Association for Computing Machinery, New York, NY, USA, Article Paper 632, 10 pages. DOI :

http://dx.doi.org/10.1145/3290605.3300862

[29] Conor O'Quigley, Mathilde Sabourin, Shirley Coyle, James Connolly, Joan Condall, Kevin Curran, Brian Corcoran, and Dermot Diamond. 2014. Characteristics of a Piezo-Resistive Fabric Stretch Sensor Glove for Home-Monitoring of Rheumatoid Arthritis. In Proceedings of the 2014 11th International Conference on Wearable and Implantable Body Sensor Networks
Workshops (BSNWORKSHOPS '14). IEEE Computer

Society, Washington, DC, USA, 23-26. DOI :

http://dx.doi.org/10.1109/BSN. Workshops.2014.15

[30] Patrick Parzer, Florian Perteneder, Kathrin Probst, Christian Rendl, Joanne Leong, Sarah Schuetz, Anita Vogl, Reinhard Schwoediauer, Martin Kaltenbrunner, Siegfried Bauer, and others. 2018. RESi: A Highly Flexible, Pressure-Sensitive, Imperceptible Textile Interface Based on Resistive Yarns. In The 31st Annual ACM Symposium on User Interface Software and Technology. ACM, 745-756.

[31] Patrick Parzer, Adwait Sharma, Anita Vogl, Jürgen Steimle, Alex Olwal, and Michael Haller. 2017. SmartSleeve: Real-time Sensing of Surface and Deformation Gestures on Flexible, Interactive Textiles, using a Hybrid Gesture Detection Pipeline Patrick. In Proceedings of the 30th Annual ACM Symposium on User Interface Software and Technology - UIST '17. 565-577. DOI :

http://dx.doi.org/10.1145/3126594.3126652

[32] E. Rehmi Post and Margaret Orth. 1997. Smart Fabric, or "Wearable Clothing". In Proceedings of the 1st IEEE International Symposium on Wearable Computers (ISWC '97). IEEE Computer Society, Washington, DC, USA, 167-.

http://dl.acm.org/citation.cfm?id=851036.856432

[33] I. Ropio, A.C. Baptista, J.P. Nobre, J. Correia, F. Belo, S. Taborda, B.M. Morais Faustino, J.P. Borges, A. Kovalenko, and I. Ferreira. 2018. Cellulose paper functionalised with polypyrrole and poly(3,4-ethylenedioxythiophene) for paper battery electrodes. Organic Electronics 62 (nov 2018), 530-535. DOI : http://dx.doi.org/10.1016/J.0RGEL . 2018.06.025

[34] Ilya Rosenberg and Ken Perlin. 2009. The UnMousePad: An Interpolating Multi-touch Force-sensing Input Pad. ACM Trans. Graph. 28, 3, Article 65 (July 2009), 9 pages. DOI : http://dx.doi.org/10.1145/1531326.1531371

[35] Valkyrie Savage, Xiaohan Zhang, and Björn Hartmann. 2012. Midas: fabricating custom capacitive touch sensors to prototype interactive objects. In Proceedings of the 25th annual ACM symposium on User interface software and technology. ACM, 579-588.

[36] Andreas Schlegel and Cedric Honnet. 2017. Digital Oxymorons: From Ordinary to Expressive Objects Using Tiny Wireless IMUs. In Proceedings of the 4th International Conference on Movement Computing (MOCO '17). ACM, New York, NY, USA, Article 4, 8 pages. DOI : http://dx.doi.org/10.1145/3077981.3078040

[37] Stefan Schneegass and Alexandra Voit. 2016. GestureSleeve: using touch sensitive fabrics for gestural input on the forearm for controlling smartwatches. Proceedings of the 2016 ACM International Symposium on Wearable Computers - ISWC'16 (2016), 108-115. DOI : http://dx.doi.org/10.1145/2971763.2971797 
[38] Sophie Skach, Patrick Healey, and Rebecca Stewart. 2017. Talking Through Your Arse: Sensing Conversation with Seat Covers.

[39] Sophie Skach, Anna Xambó, Luca Turchet, Ariane Stolfi, Rebecca Stewart, and Mathieu Barthet. 2018. Embodied Interactions with E-Textiles and the Internet of Sounds for Performing Arts. In Proceedings of the Twelfth International Conference on Tangible, Embedded, and Embodied Interaction (TEI'18). ACM, New York, NY, USA, 80-87. DOI : http://dx.doi.org/10.1145/3173225.3173272

[40] Paul Strohmeier, Jarrod Knibbe, Sebastian Boring, and Kasper Hornbæk. 2018. zPatch: Hybrid resistive/capacitive etextile input. In TEI 2018 Proceedings of the 12th International Conference on Tangible, Embedded, and Embodied Interaction, Vol. 2018-Janua. DOI :

http://dx.doi.org/10.1145/3173225.3173242

[41] Paul Strohmeier, Kaja Vembe Swensen, Cameron Lapp, Audrey Girouard, and Roel Vertegaal. 2012a. A Flock of Birds: Bringing Paper to Life. In Proceedings of the Sixth International Conference on Tangible, Embedded and Embodied Interaction (TEI'12). ACM, New York, NY, USA, 333-334. DOI : http://dx.doi.org/10.1145/2148131.2148208

[42] Paul Strohmeier, Roel Vertegaal, and Audrey Girouard. 2012b. With a Flick of the Wrist: Stretch Sensors As Lightweight Input for Mobile Devices. In Proceedings of the Sixth International Conference on Tangible, Embedded and Embodied Interaction (TEI '12). ACM, New York, NY, USA, 307-308. DOI :

http://dx.doi.org/10.1145/2148131.2148195

[43] Aneesh P. Tarun and Peng Wang. 2012. Designing and Building Inexpensive Flexible Circuits. In Proceedings of the Sixth International Conference on Tangible, Embedded and Embodied Interaction (TEI '12). ACM, New York, NY, USA, 375-377. DOI : http://dx.doi.org/10.1145/2148131.2148221

[44] Marc Teyssier, Gilles Bailly, Catherine Pelachaud, Eric Lecolinet, Andrew Conn, and Anne Roudaut. 2019. Skin-On Interfaces: A Bio-Driven Approach for Artificial Skin Design to Cover Interactive Devices. (2019). http://dx.doi.org/10.475/123_4

[45] Martin Weigel, Tong Lu, Gilles Bailly, Antti Oulasvirta, Carmel Majidi, and Jürgen Steimle. 2015. iSkin: Flexible, Stretchable and Visually Customizable
On-Body Touch Sensors for Mobile Computing. In Proceedings of the 33rd Annual ACM Conference on Human Factors in Computing Systems (CHI '15). ACM, New York, NY, USA, 2991-3000. DOI :

http://dx.doi.org/10.1145/2702123.2702391

[46] Martin Weigel, Aditya Shekhar Nittala, Alex Olwal, and Jürgen Steimle. 2017. SkinMarks: Enabling Interactions on Body Landmarks Using Conformal Skin Electronics. In Proceedings of the 2017 CHI Conference on Human Factors in Computing Systems (CHI'17). Association for Computing Machinery, New York, NY, USA, 3095-3105. DOI :

http://dx.doi.org/10.1145/3025453.3025704

[47] Michael Wessely, Theophanis Tsandilas, and Wendy E. Mackay. 2016. Stretchis: Fabricating Highly Stretchable User Interfaces. In Proceedings of the 29th Annual Symposium on User Interface Software and Technology (UIST '16). ACM, New York, NY, USA, 697-704. DOI : http://dx.doi.org/10.1145/2984511.2984521

[48] Anusha Withana, Daniel Groeger, and Jürgen Steimle. 2018. Tacttoo: A thin and feel-through tattoo for on-skin tactile output. In The 31st Annual ACM Symposium on User Interface Software and Technology. ACM, 365-378.

[49] Juan Xie, Wei Pan, Zheng Guo, Shan Shan Jiao, and Ling Ping Yang. 2019. In situ polymerization of polypyrrole on cotton fabrics as flexible electrothermal materials. Journal of Engineered Fibers and Fabrics 14 (jan 2019), 155892501982744. DOI :

http://dx.doi.org/10.1177/1558925019827447

[50] Yang Zhang, Gierad Laput, and Chris Harrison. 2017. Electrick: Low-cost touch sensing using electric field tomography. In Proceedings of the 2017 CHI Conference on Human Factors in Computing Systems. ACM, 1-14.

[51] Yang Zhang, Chouchang Jack Yang, Scott E Hudson, Chris Harrison, and Alanson Sample. 2018. Wall++: Room-scale interactive and context-aware sensing. In Proceedings of the 2018 CHI Conference on Human Factors in Computing Systems. ACM, 273.

[52] Bo Zhou, Jingyuan Cheng, Mathias Sundholm, and Paul Lukowicz. 2014. From smart clothing to smart table cloth: Design and implementation of a large scale, textile pressure matrix sensor. In Lecture Notes in Computer Science, Vol. 8350 LNCS. Springer, Cham, 159-170. DOI :

http://dx.doi.org/10.1007/978-3-319-04891-8_14 